\title{
A Multiple Beamforming Network for Unequally Spaced Linear Array Based on CORPS
}

\author{
Armando Arce, ${ }^{1}$ Marco Cardenas-Juarez, ${ }^{1}$ Ulises Pineda-Rico, ${ }^{1}$ \\ David H. Covarrubias, ${ }^{2}$ and Enrique Stevens-Navarro ${ }^{1}$ \\ ${ }^{1}$ Faculty of Sciences, Universidad Autonoma de San Luis Potosi, 78290 San Luis Potosi, SLP, Mexico \\ ${ }^{2}$ Department of Electronics and Telecommunications, CICESE Research Centre, 22860 Ensenada, BC, Mexico \\ Correspondence should be addressed to Enrique Stevens-Navarro; estevens@galia.fc.uaslp.mx
}

Received 11 May 2015; Revised 26 June 2015; Accepted 1 July 2015

Academic Editor: Quanyuan Feng

Copyright (C) 2015 Armando Arce et al. This is an open access article distributed under the Creative Commons Attribution License, which permits unrestricted use, distribution, and reproduction in any medium, provided the original work is properly cited.

\begin{abstract}
This paper proposes an alternative and innovative way to design a simpler beamforming network (BFN) based on balancing alternated power combiners and dividers, to feed a nonuniformly spaced linear array with Gaussian amplitude and coherent (inphase) signals. Thus, a two-beam design configuration of the feeding network for a nonuniform array with beam steering capability is proposed and analyzed. The nonuniform aperture and the complex inputs of the feeding network are optimized by means of a differential evolution algorithm. In addition, a comparative analysis between a uniform and nonuniform linear array with the proposed feeding network is performed. Simulation results show the advantages and effectiveness of the proposed feeding network exploiting the nonuniformity of the antenna elements, in terms of side lobe level and directivity. Furthermore, research results show an inherent reduction in hardware complexity of the network.
\end{abstract}

\section{Introduction}

Beamforming networks for multiple-beam antennas are of great interest due to their performance and flexibility in many application fields including terrestrial and space telecommunications [1]. In general beamforming networks provide predefined excitation vectors (amplitude and phase) to an antenna array to generate a radiation pattern of certain desired design [2]. Multiple beamforming networks have been studied for decades, with Butler, Blass, and Nolen being the most outstanding matrices [3-5]. As a result of feeding the input ports, the outputs in these lossless multiport beamforming networks are orthogonal, which is demonstrated mathematically by its inner product. However, in these lossless feeding networks the orthogonal excitation laws impose severe restrictions on the beam shape, which implies a rigorous optimization design [6].

This paper proposes an alternative solution based on a simpler design approach where only power combiners and dividers are used. This coherent (in-phase) network introduced in [7] is known as coherently radiating periodic structure beamforming network (C-BFN or CORPS-BFN) and generates an in-phase Gaussian-like amplitude distribution. Recent research work based on C-BFN reported approaches for simplifying the network hardware in different antenna geometries, the development of new structures to improve efficiency, and practical implementation of the feeding network [8-11]. However, the impact of using nonuniformly spaced arrays in C-BFN has not been analyzed previously in the literature.

In contrast, this work presents a deeper insight on the performance of a C-BFN that feeds a nonuniformly spaced linear array. Then, the objective is to propose a C-BFN for an unequally spaced linear array using differential evolution (DE) technique to optimize the required complex excitations at the input ports and the spacing between antenna elements, thus obtaining a multibeam and nonuniformly spaced linear array with beam steering capabilities. To demonstrate the performance of a nonuniformly spaced linear array, a comparative analysis with a uniform linear array in terms of side lobe level and directivity is performed. This latter is supported by simulation results. 


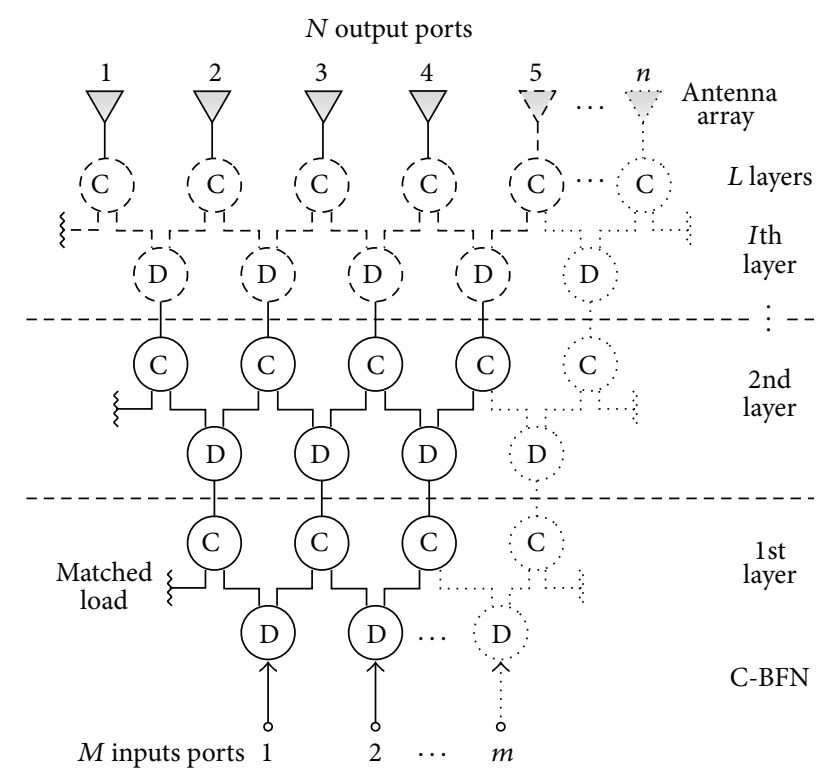

FIGURE 1: Schematic of a general topology of a C-BFN with power dividers (D) and power combiners (C) nodes of $M$ inputs, $N$ outputs, and $L$ layers.

The paper is organized as follows. Section 2 describes briefly the operation of a general C-BFN and the optimization strategy followed. Section 3 presents the simulation setup and the obtained results with a description of the proposed two-beam configuration. Finally, conclusions of this work are presented in Section 4.

\section{Theoretical Study}

2.1. C-BFN Model. The conceptual model of C-BFN is an interconnection of successive layers with a specific arrangement of alternating an identical basic node to create an entire feed network, as described in [7]. The main characteristics of the network allow controlling the radiation patterns, including side lobes and main lobe shapes with stable and independent beam pointing. The basic node can act as a power divider (D) or power combiner (C) depending on its position as illustrated in Figure 1. The behavior of C-BFN is based on a binomial excitation law for a linear array, where the coefficients can be obtained by Pascal's rule (binomial expansion) [12]. The basic constituting component can be characterized as a 3-port component (for power combination and power division) by the following Wilkinson's matrix [7]:

$$
[S]_{\text {node }}=\frac{j}{\sqrt{2}}\left[\begin{array}{lll}
0 & 1 & 1 \\
1 & 0 & 0 \\
1 & 0 & 0
\end{array}\right] .
$$

It is important to point out that the ideal matrix in (1) ensures that the 3-port component has no interaction between the input signals since it is perfectly coupled and isolated.

As shown in Figure 1, each layer is an arrangement of basic nodes; $\mathrm{C}$ nodes on each layer are connected to the
$\mathrm{D}$ nodes on the next layer. The output of C-BFN generates a Gaussian-like amplitude distribution and in-phase (true time delay) excitation law in the $m$ th input ports. This basic topology makes it feasible to establish several configurations with different inputs, outputs, and layers for mono- and multibeam designs. In general, the number of output ports $(N)$ in C-BFN is greater than the number of input ports $(M)$ and the number of layers is determined by $N-M$. In order to calculate the output of a C-BFN for a given linear array, we can use [10]

$$
\left[b_{m+1}\right]_{(m+1, m)}=\left[T_{m}\right]_{(m+1, m)} \cdot\left[a_{m}\right]_{(m, 1)},
$$

where $\left[T_{m}\right]$ denotes a transfer matrix per layer and $\left[b_{m+1}\right]$ and $\left[a_{m}\right]$ are the output and input vectors, respectively. Subscripts in brackets indicate the size of the matrices where index $m$ is equal to the number of input ports.

The transfer matrix can be defined based on the transfer coefficients of the basic node as given in (1). Hence, the general matrix form is expressed as

$$
\left[T_{m}\right]=\frac{1}{2}\left[\begin{array}{ccccc}
\sqrt{2} & 0 & 0 & \cdots & 0 \\
1 & 1 & 0 & \cdots & 0 \\
0 & 1 & 1 & & \vdots \\
0 & 0 & \ddots & \ddots & 0 \\
\vdots & \vdots & & 1 & 1 \\
0 & 0 & \cdots & 0 & \sqrt{2}
\end{array}\right]_{m+1, m}
$$

Thus, evaluating (2) and interconnecting a feed network, as in Figure 1, make it possible to simulate and analyze a CBFN. Such simulation can be performed using an iterative code (e.g., in MATLAB) that models the propagation of signals throughout a general C-BFN. Additionally, this multimode network could be physically implemented replicating this basic constituting component, for example, by means of a circular in-phase hybrid ring for wideband performances [11].

Let us now consider a linear geometry of the antenna array at the top of the BFN with $N$ omnidirectional antenna elements in the azimuth plane ( $x y$-plane). The array factor for a set of arbitrary complex inputs [I] feeding a C-BFN with arbitrary antenna element positions $[\mathbf{x}]$ is derived from [12] and given by

$$
\operatorname{AF}(\theta, \mathbf{I}, \mathbf{x})=\sum_{n=1}^{N} I_{n} e^{j\left(k x_{n} \cos \theta-\alpha_{n}\right)},
$$

where $I_{n}$ represents the complex weights $\left(I_{n}=A_{n} e^{j \xi_{n}}\right)$ at the $n$th input port of the feeding network, $k$ is the wavenumber, $x_{n}$ is the location of the $n$th element, and $\alpha_{n}=k x_{n} \cos \theta_{0}$ is a phase delay related to the maximum directivity attained $\left(\theta_{0}\right)$.

Furthermore, the antenna synthesis is optimized using an evolutionary algorithm which handles several combinations of possible inputs to find a near-optimal solution for specific beam shaping objectives. In this context, stochastic optimization algorithms are recommended for nonlinear and 


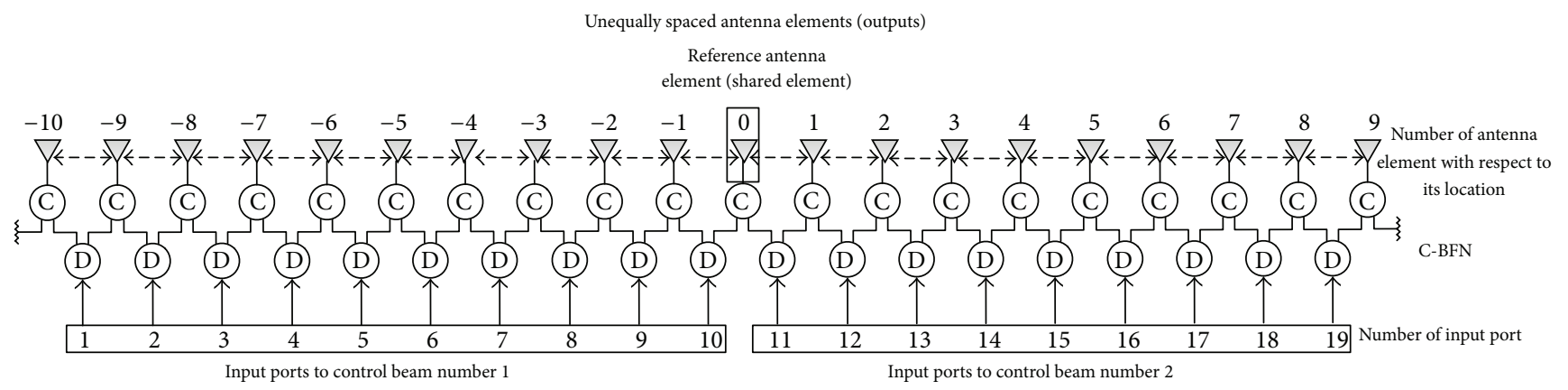

Figure 2: Schematic of the two-beam configuration showing the C-BFN and its interconnection with the nonuniformly spaced linear array.

nonconvex optimization problems [13]. The optimization method to obtain near-optimal solutions is described below.

2.2. C-BFN Optimization Process. Differential evolution is the optimization strategy used in the C-BFN system to obtain complex excitations (amplitudes and phases) and antenna element locations. DE is a well-known population-based metaheuristic widely used for beam pattern synthesis [14]. In DE the set of parameters to be optimized define an agent or potential solution. A collection of "vectors" or agents form the population where some of these agents are extracted and geometrically manipulated. The main actor in the behavior of $\mathrm{DE}$ is performed by the mutation operator, where, for each vector or agent $\left(\varepsilon_{i}^{G} \mid i=1,2, \ldots, N_{p}\right)$ in the population $\left(N_{p}\right)$ of the Gth iteration, a new donor vector $\left(\varepsilon_{d}\right)$ is generated as follows:

$$
\varepsilon_{d}=\varepsilon_{r 1}^{G}+F\left(\varepsilon_{r 2}^{G}-\varepsilon_{r 3}^{G}\right)
$$

where $r_{1}, r_{2}, r_{3} \in\left[1, N_{p}\right]$ are random integers chosen such that $r_{1} \neq r_{2} \neq r_{3}$ and $F \in[0,2]$ is the differential weight or mutation factor, that is, a real constant factor which controls the amplification of the differential variation $\left(\varepsilon_{r 2}^{G}-\varepsilon_{r 3}^{G}\right)$.

The optimization process followed in this work is sequentially described below: DE generates random vectors or agents as possible solutions. These agents are encoded by a vector of complex numbers (amplitudes and phases) $\mathbf{I}=$ $\left[A_{1} e^{j \xi_{1}}, A_{2} e^{j \xi_{2}}, \ldots, A_{M} e^{j \xi_{M}}\right]$ and a vector of real numbers representing antenna element positions $\mathbf{x}=\left[x_{1}, x_{2}, \ldots, x_{N}\right]$. Thus, each agent proposes a radiation pattern of certain performance of side lobe level and directivity in each beam. Then, the mutation operator (5) in DE expands the search space, followed by the crossover and selection operators, respectively. The crossover parameter controls the number of components inherited from the mutation operator with certain crossover probability called crossover rate (CR), influencing the convergence speed. Finally, the algorithm finds a global solution generating a beam pattern with minimum side lobe level and maximum directivity in a predefined direction for each beam. To achieve the above goal the following optimization problem is formulated and applied for each beam:

$$
\begin{array}{cc}
\underset{\mathbf{I}, \mathbf{x}}{\operatorname{minimize}} & \left(\frac{\left|\mathrm{AF}\left(\theta_{\mathrm{SLL}}, \mathbf{I}, \mathbf{x}\right)\right|}{\max |\operatorname{AF}(\theta, \mathbf{I}, \mathbf{x})|}\right)+\left(\frac{1}{\mathrm{D}(\theta, \mathbf{I}, \mathbf{x})}\right) \\
\text { subject to } \quad \mathbf{I}=\left\{A_{m} e^{j \xi_{m}} \mid A_{m} \in \mathbb{A}, \quad \xi_{m} \in \mathbb{P}\right\}, \\
\forall m=[1,2, \ldots, M], \\
\quad \mathbf{x}=\left\{x_{n} \mid x_{n} \in \mathbb{X}\right\}, \quad \forall n=[1,2, \ldots, N],
\end{array}
$$

where $\mathbb{X}$ is the antenna element location domain, $\mathbb{A}$ and $\mathbb{P}$ are the amplitude and phase domains, respectively, and $\theta_{\mathrm{SLL}}$ is the angle where the maximum side lobe is attained. The goal is to minimize the weighted sum that involves both side lobe level and directivity, respectively, which are uniformly weighted in the cost function. Detailed information of the DE procedure can be found in [15-17].

\section{Simulation Setup and Results}

For assessing and analyzing the effect of a C-BFN in a nonuniform linear array (NULA), a two-beam design was implemented in MATLAB and is described as follows.

The proposed configuration is shown in Figure 2; the schematic represents a nonuniformly spaced linear array of 20 antenna elements with 19 feeding ports with a C-BFN of one layer. In this multimode network, 10 input ports are fed to control 11 output ports directly connected to the same number of antennas generating beam number 1. Similarly, 9 input ports are excited to manipulate 10 antenna elements, where the total output corresponds to beam number 2 . With this network topology, based on subsets of input ports, two orthogonal beams can be generated sharing an antenna element. This shared antenna element is used as reference (i.e., a fixed antenna element) for the location of the other antenna elements. Therefore, the unequally spaced locations of the antenna elements take values either to the left or right. The first beam is shaped and controlled by the first ten of the total feeding ports (19 input ports), and the 9 remaining input ports are used to conform the other beam. 


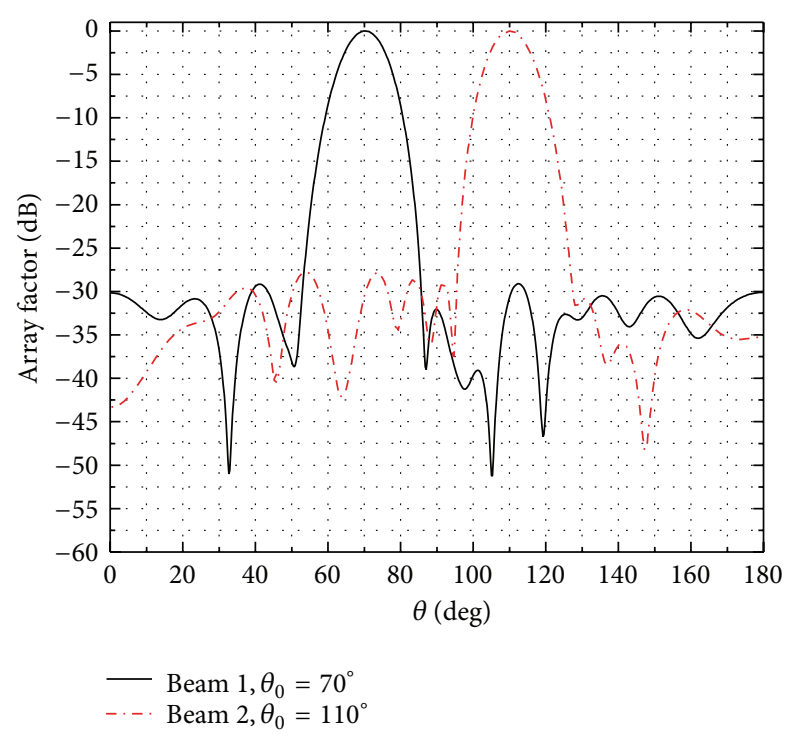

FIGURE 3: Radiation patterns of the two-beam configuration of CBFN with a nonuniform linear array optimized by DE with beam steering in $70^{\circ}$ and $110^{\circ}$.

In this two-beam configuration under study, the objective is to assess the radiation pattern generated by the C-BFN in an unequally spaced linear array. Furthermore, the results are compared with the same configuration of C-BFN but in a uniform linear array (ULA) with the purpose of comparing performance.

The DE algorithm is programmed for the assessment of array factor. Due to the algorithm stochasticity we execute the algorithm 20 times for each direction of interest in each beam. The array factor considers a steering range of $80^{\circ}$ with an angular step of $10^{\circ}$ for a nonuniform linear array of 20 antenna elements with random nonuniform spacing between $d_{\min }=0.5 \lambda$ (wavelength) and $d_{\max }=1 \lambda$. For the uniform case $d=0.5 \lambda$. The parameters of the $\mathrm{DE}$ algorithm were set as follows: the algorithm is executed using $N_{p}=500$ individuals (population size) through 500 iterations to ensure a good sampling of the solution space, with a crossover probability $\mathrm{CR}=1.0$ and a mutation factor set in $F=0.5$. A DE scheme with random selection, one difference vector, and binomial crossover was implemented $[16,17]$.

3.1. Simulation Results. Figure 3 illustrates the resulting normalized radiation pattern of the two-beam configuration generated by the feeding network detailed in the previous section. The complex excitations at the input ports of the CBFN along with the antenna locations were optimized with DE (see Table 1), providing a good performance in terms of side love level and directivity. The optimized radiation patterns in this proposal demonstrate the ability of the network to handle side lobe level (SLL $\leq-27 \mathrm{~dB}$ ) and directivity in different beam steering directions for each of the two beams. Table 1 shows the specific values in amplitude and phase distributions, and element locations for the array factors generated by the two-beam design of C-BFN and
TABLE 1: Amplitude and phase distributions and element locations for the two-beam configuration shown in Figure 3.

\begin{tabular}{lcc}
\hline \multicolumn{2}{c}{ Input ports $m=19$} & Output ports $n=20$ \\
Amplitude & $\begin{array}{c}\text { Phase } \\
\text { distribution }\end{array}$ & Element location $\left(\lambda_{n}\right)$ \\
\hline 4.02 & 48.34 & -5.4662 \\
5.02 & 3.66 & -4.9659 \\
5.74 & -4.89 & -4.3838 \\
8.02 & 8.41 & -3.8637 \\
9.76 & 5.37 & -3.2990 \\
9.95 & 7.11 & -2.7913 \\
8.69 & 8.51 & -2.2536 \\
7.20 & 11.10 & -1.7234 \\
6.04 & 8.21 & -1.1825 \\
4.66 & -21.01 & -0.5800 \\
4.82 & -8.71 & 0 \\
6.40 & 14.71 & 0.5118 \\
7.04 & 11.95 & 1.0857 \\
9.39 & 13.23 & 1.5877 \\
9.17 & 0.19 & 2.1078 \\
11.89 & 1.10 & 2.6123 \\
11.01 & 2.29 & 3.1320 \\
8.03 & 2.03 & 3.6639 \\
6.49 & 8.10 & 4.2142 \\
- & - & 4.7562 \\
\hline & &
\end{tabular}

illustrated in Figure 3. The table provides the solution to generate each beam pattern considering specific directions of maximum radiation previously defined.

The direction of maximum gain in this specific multibeam case is scanned along the azimuth plane ( $\theta$-axis) and set in $\theta_{0}=70^{\circ}$ for beam 1 and $\theta_{0}=110^{\circ}$ for beam 2 . In addition, in this particular C-BFN, the control of each beam is based on subsets of consecutive input ports. In this topology of a two-beam C-BFN, the feeding network of one layer can scan each main beam to any desired location by using $N-1$ input ports for each beam. In other words, a C-BFN of one stage as shown in Figure 2 reduces the internal circuitry of a conventional phased antenna array with direct feeding. This implies that the network reduces two input ports and one antenna element because it uses 10 input ports for controlling 11 antenna elements for beam number 1. Similarly, it uses only 9 input ports for controlling 10 antenna elements for beam number 2. In this case, the proposed configuration allows the reuse of one antenna element located between input ports numbers 10 and 11 (see Figure 2); this is possible by the basic principles of operation of the C-BFN.

To develop a comprehensive and fair comparative analysis of the linear array system that considers a C-BFN, the behavior of the array factor is analyzed by means of the CBFN shown in Figure 2, for a uniform and nonuniform linear array. Each configuration is previously optimized by DE with the parameter settings described in Section 3. To demonstrate the potential benefits of nonuniform linear array over 


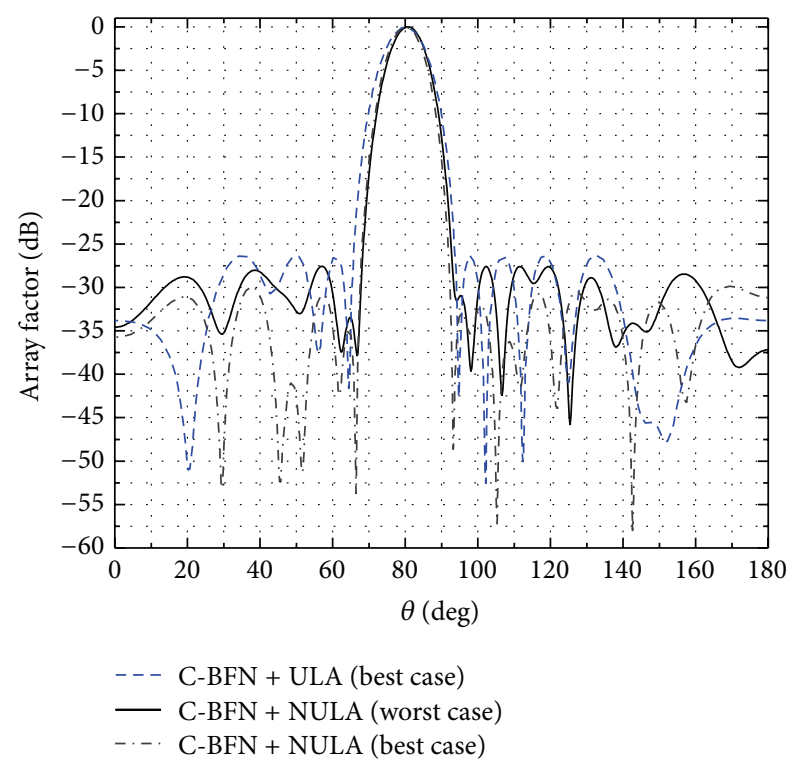

FIGURE 4: Comparison between the array factor of the best case of a ULA, the worst case of a NULA, and the best case of a NULA obtained by the proposed C-BFN, showing an example of beam \#1 directed at $80^{\circ}$.

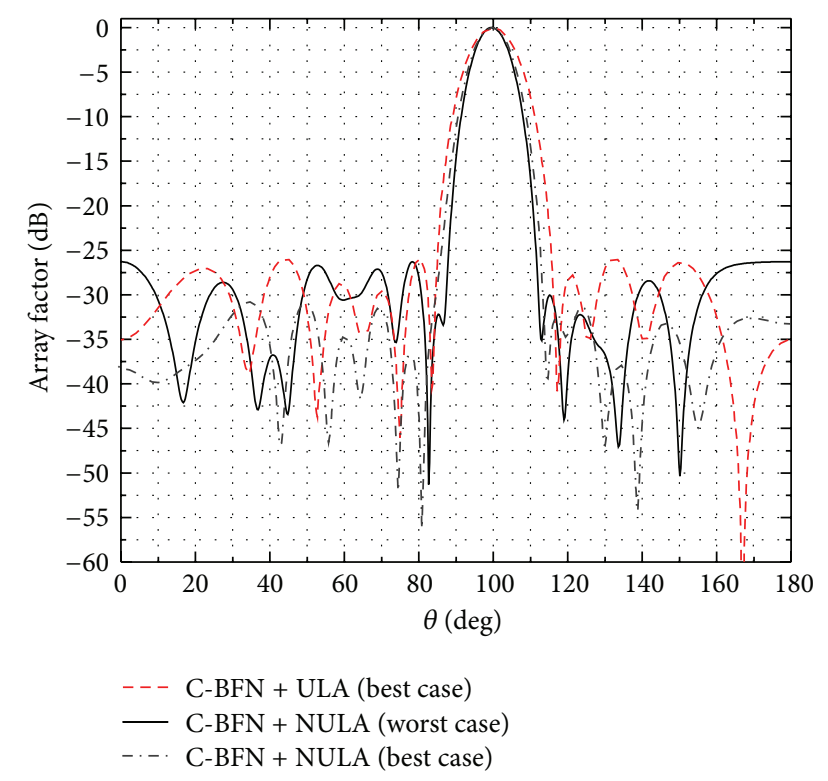

FIGURE 5: Comparison between the array factor of the best case of a ULA, the worst case of a NULA, and the best case of a NULA obtained by the proposed C-BFN, showing an example of beam \#2 directed at $100^{\circ}$.

a uniform linear array in a C-BFN, we compare the worst result of a NULA with the best result of a ULA, and additionally with best case of a NULA with the same proposed C-BFN configuration under equal conditions and several trials.

Figures 4 and 5 show an example of the radiation pattern generated by the proposed two-beam configuration of C-BFN for equally and unequally spaced linear array. Specifically, Figure 4 shows beam number 1 , set in $\theta_{0}=80^{\circ}$ for both linear configurations (i.e., uniform and nonuniform). In this example, the main beam is conformed by 10 input ports controlling 11 antenna elements, in all cases. The specific values of SLL and directivity (DIR) are $-26.27 \mathrm{~dB}$ and $9.73 \mathrm{~dB}$, respectively, for the best result of a $\mathrm{C}-\mathrm{BFN}$ in a uniform linear array (C-BFN + ULA (best case)), $-27.55 \mathrm{~dB}$ and $10.47 \mathrm{~dB}$ in the worst result of a C-BFN using a nonuniform linear array (C-BFN + NULA (worst case)), and $-29.86 \mathrm{~dB}$ and $10.57 \mathrm{~dB}$ for the best result of a $\mathrm{C}-\mathrm{BFN}$ in a nonuniform linear array (C-BFN + NULA (best case)). In the same way, Figure 5 illustrates the performance of beam number 2 that is set in $\theta_{0}=100^{\circ}$ for the three configurations. In this figure, the best array factor of a CBFN + ULA exhibits $-25.87 \mathrm{~dB}$ and $9.32 \mathrm{~dB}$ of SLL and DIR, respectively, $-26.27 \mathrm{~dB}$ and $10.56 \mathrm{~dB}$ in the worst array factor of a CBFN + NULA, and $-30.78 \mathrm{~dB}$ and $10.21 \mathrm{~dB}$ for the best array factor of a CBFN + NULA. Beam number 2 is conformed using only 9 input ports to control 10 input ports. Despite the fact that beam number 2 is conformed with a smaller antenna aperture, it obtains a similar performance as beam number 1 . This is due to the fact that the use of one more antenna may not represent a significant performance advantage in this configuration. However, in a physical implementation of the complete antenna system the type of antenna used could represent a more relevant performance. As can be seen in Figures 4 and 5, the performance of the worst array factor in a CBFN + NULA is slightly better than the best radiation pattern in a CBFN + ULA, even over all the steering range (as shown in Tables 2 and 3). This behavior demonstrates that a C-BFN based on a nonuniform linear array outperforms in equal conditions a C-BFN based on uniform linear array, in terms of side lobe level and directivity. In the same way, we can observe in Figures 4 and 5 a marked superiority in performance of the best case of C-BFN + NULA over the best case of C-BFN + ULA with an advantage in average of $4 \mathrm{~dB}$ of SLL and $1 \mathrm{~dB}$ of DIR for the two beams, including different directions of interest (see Tables 2 and 3 ).

Next, numerical values of SLL and DIR for the generated radiation patterns (beams numbers 1 and 2) with the proposed configuration in both cases, ULA and NULA, are presented in Tables 2 and 3. These two tables collect information of the best numerical values of side lobe level and directivity obtained by the uniform linear array and the worst and best numerical values of the aforementioned metrics obtained by the nonuniformly spaced linear array. In general, a C-BFN with a uniform or nonuniform linear system retains fundamental properties of linear arrays, with decreasing values SLL and DIR as we move away from the broadside region. As it was previously shown in Figures 4 and 5 , the comparative analysis between linear arrays allows us to ensure that a C-BFN with a nonuniform linear array has better performance than a C-BFN with uniform linear array in terms of directivity and side lobe level.

Although the focus in this work is on a two-beam configuration, it is possible to implement different C-BFN configurations by varying the number of output and input ports based on the general topology and beams required. In this design approach for an unequally spaced linear array based on C-BFN, it is important to note the technological 
TABLE 2: Numerical values of side lobe level and directivity for the multiple beamforming network with a uniform and nonuniform linear array for beam number 1 .

\begin{tabular}{|c|c|c|c|c|c|c|}
\hline \multirow{2}{*}{$\begin{array}{l}\text { Steering range } \\
\theta_{0}(\mathrm{deg})\end{array}$} & \multicolumn{2}{|c|}{ C-BFN + ULA best case } & \multicolumn{2}{|c|}{ C-BFN + NULA worst case } & \multicolumn{2}{|c|}{$\mathrm{C}-\mathrm{BFN}+\mathrm{NULA}$ best case } \\
\hline & SLL $(\mathrm{dB})$ & $\operatorname{DIR}(\mathrm{dB})$ & SLL (dB) & $\operatorname{DIR}(\mathrm{dB})$ & SLL (dB) & $\operatorname{DIR}(\mathrm{dB})$ \\
\hline 50 & -18.65 & 8.62 & -20.01 & 9.33 & -22.30 & 9.47 \\
\hline 60 & -22.01 & 8.59 & -23.12 & 9.52 & -26.22 & 9.44 \\
\hline 70 & -24.42 & 8.63 & -25.77 & 9.91 & -29.09 & 9.50 \\
\hline 80 & -26.27 & 9.73 & -27.55 & 10.47 & -29.86 & 10.57 \\
\hline 90 & -28.22 & 9.91 & -29.37 & 10.61 & -31.91 & 10.73 \\
\hline 100 & -25.92 & 9.89 & -27.32 & 10.24 & -29.52 & 10.79 \\
\hline 110 & -24.98 & 8.55 & -25.84 & 9.85 & -28.93 & 9.58 \\
\hline 120 & -21.95 & 8.61 & -22.96 & 9.78 & -25.97 & 9.52 \\
\hline 130 & -19.11 & 8.74 & -20.13 & 9.46 & -23.68 & 9.69 \\
\hline
\end{tabular}

TABLE 3: Numerical values of side lobe level and directivity for the multiple beamforming network with a uniform and nonuniform linear array for beam number 2 .

\begin{tabular}{|c|c|c|c|c|c|c|}
\hline \multirow{2}{*}{$\begin{array}{l}\text { Steering range } \\
\theta_{0}(\mathrm{deg})\end{array}$} & \multicolumn{2}{|c|}{ C-BFN + ULA best case } & \multicolumn{2}{|c|}{ C-BFN + NULA worst case } & \multicolumn{2}{|c|}{ C-BFN + NULA best case } \\
\hline & SLL (dB) & $\operatorname{DIR}(\mathrm{dB})$ & SLL (dB) & $\operatorname{DIR}(\mathrm{dB})$ & SLL (dB) & $\operatorname{DIR}(\mathrm{dB})$ \\
\hline 50 & -17.81 & 8.43 & -19.52 & 9.15 & -22.23 & 9.53 \\
\hline 60 & -21.33 & 8.38 & -22.16 & 9.33 & -25.32 & 9.46 \\
\hline 70 & -23.71 & 8.42 & -24.56 & 9.82 & -27.48 & 9.28 \\
\hline 80 & -25.44 & 9.67 & -25.98 & 10.72 & -29.80 & 10.65 \\
\hline 90 & -27.13 & 9.83 & -28.02 & 10.63 & -31.24 & 10.76 \\
\hline 100 & -25.87 & 9.32 & -26.27 & 10.56 & -30.78 & 10.21 \\
\hline 110 & -23.66 & 8.74 & -24.72 & 9.68 & -27.74 & 9.54 \\
\hline 120 & -20.89 & 8.42 & -21.95 & 9.52 & -24.45 & 9.36 \\
\hline 130 & -18.15 & 8.39 & -19.97 & 9.08 & -22.77 & 9.59 \\
\hline
\end{tabular}

advantages of the feeding network itself (i.e., the reuse of antenna elements and the reduction of control signals inputs) compared with a conventional linear array in a phased array (direct feeding). For the two-beam configuration, each beam is shaped with $M=N-1$ input ports reusing one antenna element (reference radiator). In general, the complexity reduction and the attained performance in different directions of interest in a nonuniformly spaced linear array using a C-BFN is a good option for several applications where conventional nonuniform linear arrays are used $[18,19]$.

\section{Conclusion}

The design of a C-BFN for a two-beam steerable configuration in an unequally spaced linear array has been presented. The design approach involved the behavior of the array factors generated by the nonuniform and uniform linear arrays in the proposed C-BFN configuration. The results of this research proved that the proposed design of a C-BFN can generate scannable and independent beams in linear arrays exploiting the nonuniformity of the antenna array with a reduced complexity of the antenna system. Furthermore, the proposed C-BFN configuration exposes the advantages of adopting this technology in unequally spaced linear arrays for applications requiring multibeams with steering capabilities in this antenna geometry.

\section{Conflict of Interests}

The authors declare that there is no conflict of interests regarding the publication of this paper.

\section{Acknowledgments}

This work was partially supported by the Mexican National Science and Technology Council (CONACyT) and Universidad Autonoma de San Luis Potosi (UASLP).

\section{References}

[1] P. Angeletti and M. Lisi, "Multimode beamforming networks for space applications," IEEE Antennas and Propagation Magazine, vol. 56, no. 1, pp. 62-78, 2014.

[2] M. Schuühler, "On evaluation of beamforming networks," IEEE Antennas and Wireless Propagation Letters, vol. 13, pp. 766-769, 2014.

[3] J. Butler and R. Lowe, "Beam-forming matrix simplifies design of electronically scanned antennas," Electronic Design, vol. 9, pp. $170-173,1961$. 
[4] J. Blass, "Multidirectional antenna-a new approach to stacked beams," in Proceedings of the 1958 IRE International Convention Record, vol. 8, pp. 48-50, New York, NY, USA, March 1966.

[5] J. Nolen, Synthesis of multiple beam networks for arbitrary illuminations [Ph.D. dissertation], Bendix Corporation, Radio Division, Baltimore, Md, USA, 1965.

[6] J. Allen, "A theoretical limitation on the formation of lossless multiple beams in linear arrays," IRE Transactions on Antennas and Propagation, vol. 9, no. 4, pp. 350-352, 1961.

[7] D. Betancourt and C. del Río Bocio, "A novel methodology to feed phased array antennas," IEEE Transactions on Antennas and Propagation, vol. 55, no. 9, pp. 2489-2494, 2007.

[8] A. Arce, L. F. Yepes, D. H. Covarrubias, and M. A. Panduro, "A new approach in the simplification of a multiple-beam forming network based on CORPS using compressive arrays," International Journal of Antennas and Propagation, vol. 2012, Article ID 251865, 8 pages, 2012.

[9] A. Arce, M. A. Panduro, D. H. Covarrubias, and A. L. Mendez, "An approach for simplifying a multiple beam-forming network for concentric ring arrays using CORPS," Journal of Electromagnetic Waves and Applications, vol. 28, no. 4, pp. 430-441, 2014.

[10] N. Ferrando and N. J. G. Fonseca, "Investigations on the efficiency of array fed coherently radiating periodic structure beam forming networks," IEEE Transactions on Antennas and Propagation, vol. 59, no. 2, pp. 493-502, 2011.

[11] N. J. G. Fonseca, "Design and implementation of a closed cylindrical BFN-fed circular array antenna for multiple-beam coverage in Azimuth," IEEE Transactions on Antennas and Propagation, vol. 60, no. 2, pp. 863-869, 2012.

[12] C. A. Balanis, Antenna Theory: Analysis and Design, John Wiley \& Sons, New York, NY, USA, 2nd edition, 1997.

[13] A. E. Eiben and J. E. Smith, Introduction to Evolutionary Computing, Springer, New York, NY, USA, 1st edition, 2003.

[14] P. Rocca, G. Oliveri, and A. Massa, "Differential evolution as applied to electromagnetics," IEEE Antennas and Propagation Magazine, vol. 53, no. 1, pp. 38-49, 2011.

[15] V. Gonuguntla, R. Mallipeddi, and K. C. Veluvolu, "Differential evolution with population and strategy parameter adaptation," Mathematical Problems in Engineering, vol. 2015, Article ID 287607, 10 pages, 2015.

[16] S. Yang, A. Qing, and Y.-B. Gan, "Synthesis of low sidelobe antenna arrays using the differential evolution algorithm," in Proceedings of the IEEE Antennas and Propagation Society International Symposium, vol. 1, pp. 780-783, IEEE, Columbus, Ohio, USA, June 2003.

[17] C. Lin, A. Qing, and Q. Feng, "Synthesis of unequally spaced antenna arrays by using differential evolution," IEEE Transactions on Antennas and Propagation, vol. 58, no. 8, pp. 2553-2561, 2010.

[18] D. G. Kurup, M. Himdi, and A. Rydberg, "Synthesis of uniform amplitude unequally spaced antenna arrays using the differential evolution algorithm," IEEE Transactions on Antennas and Propagation, vol. 51, no. 9, pp. 2210-2217, 2003.

[19] G. Toso and P. Angeletti, "Optimal combined amplitude-density synthesis of aperiodic arrays," in Proceedings of the 5th European Conference on Antennas and Propagation (EUCAP '11), pp. 3044-3047, April 2011. 

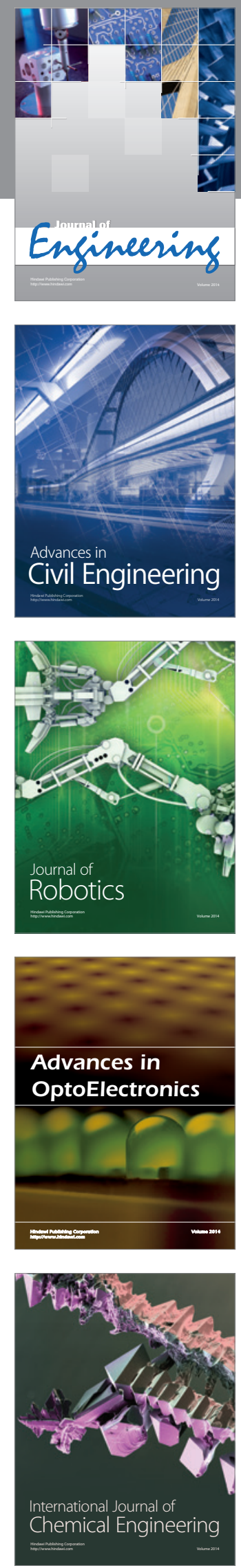

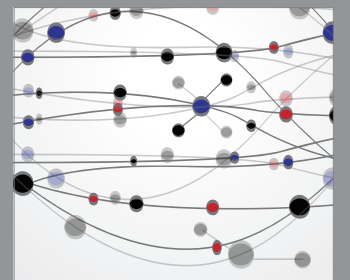

The Scientific World Journal
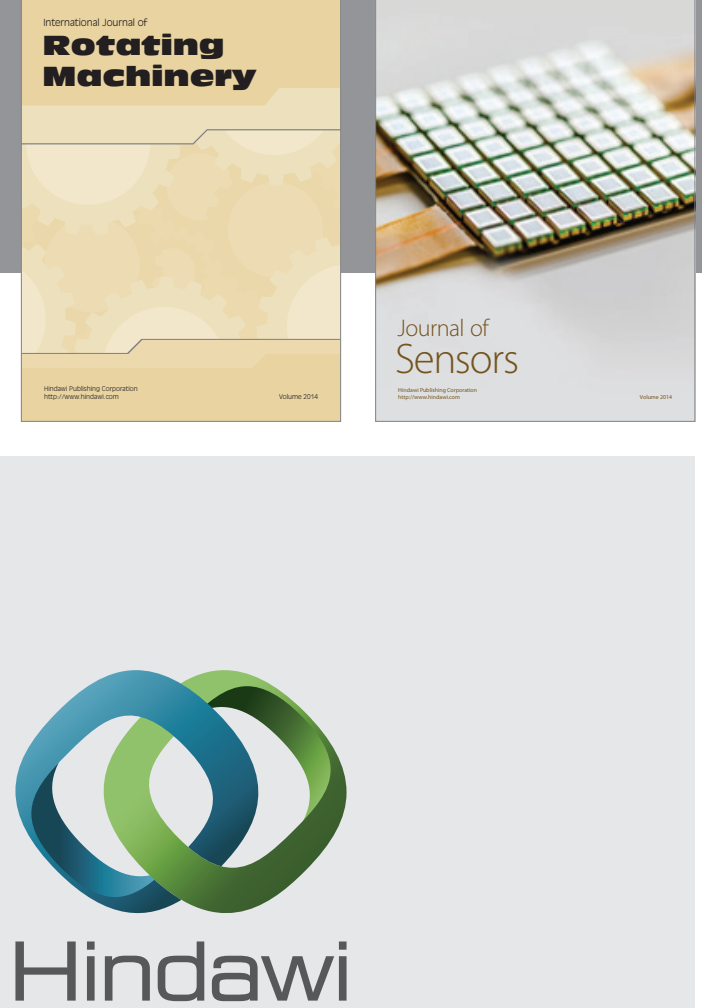

Submit your manuscripts at http://www.hindawi.com
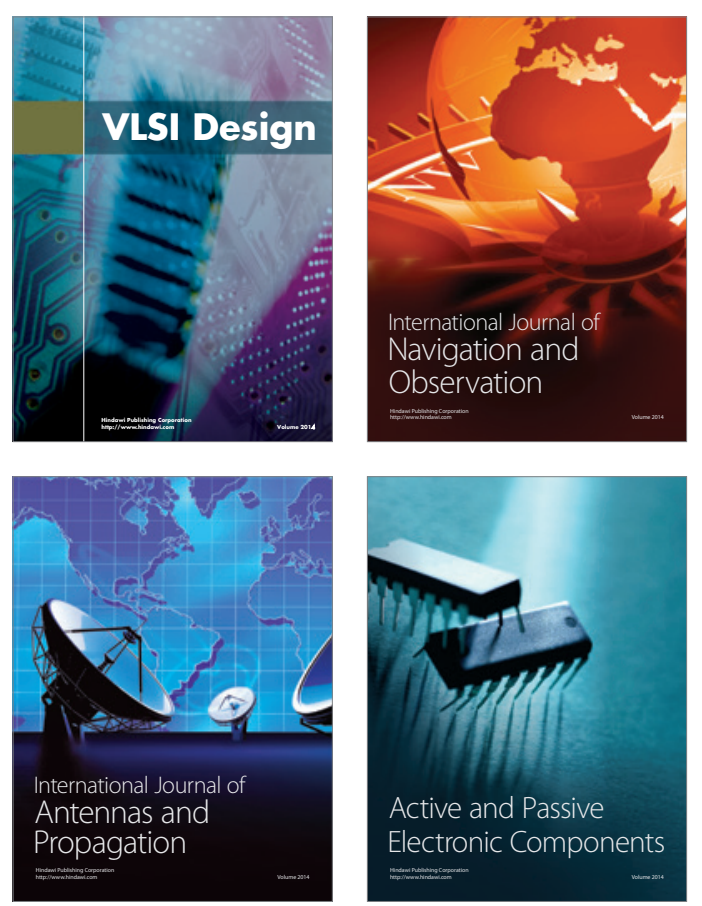
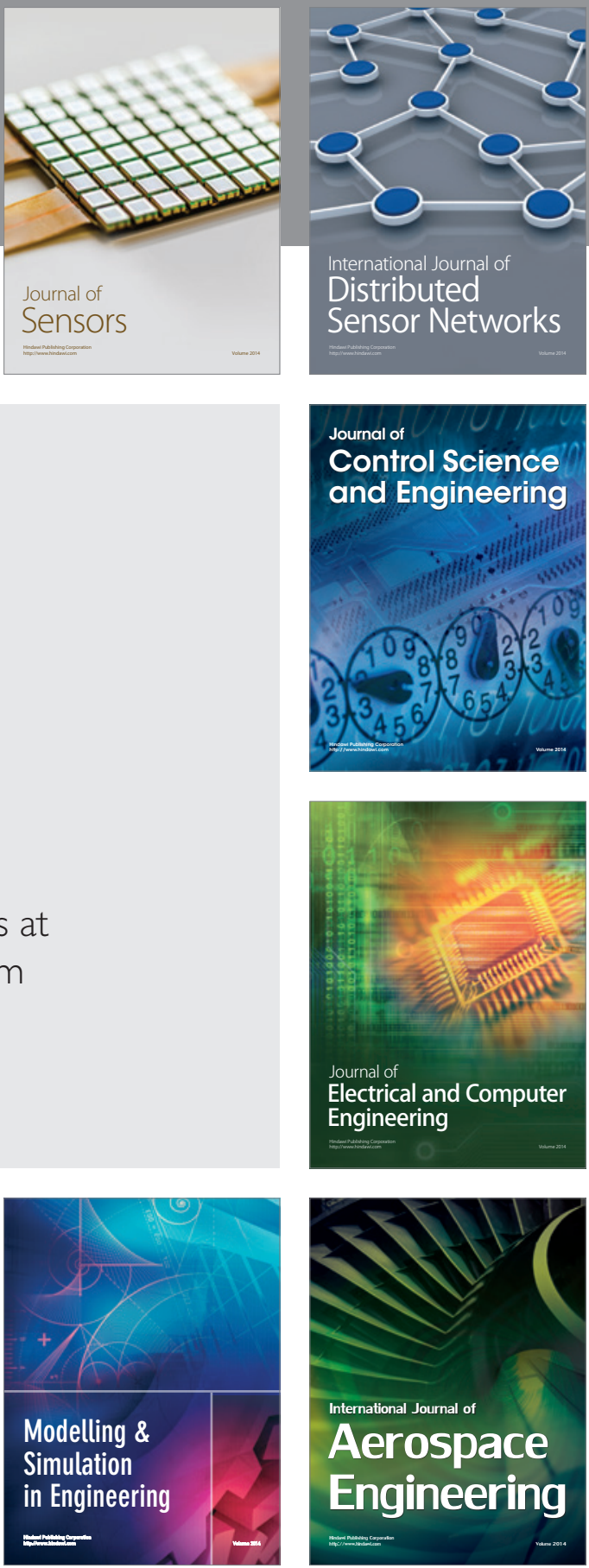

Journal of

Control Science

and Engineering
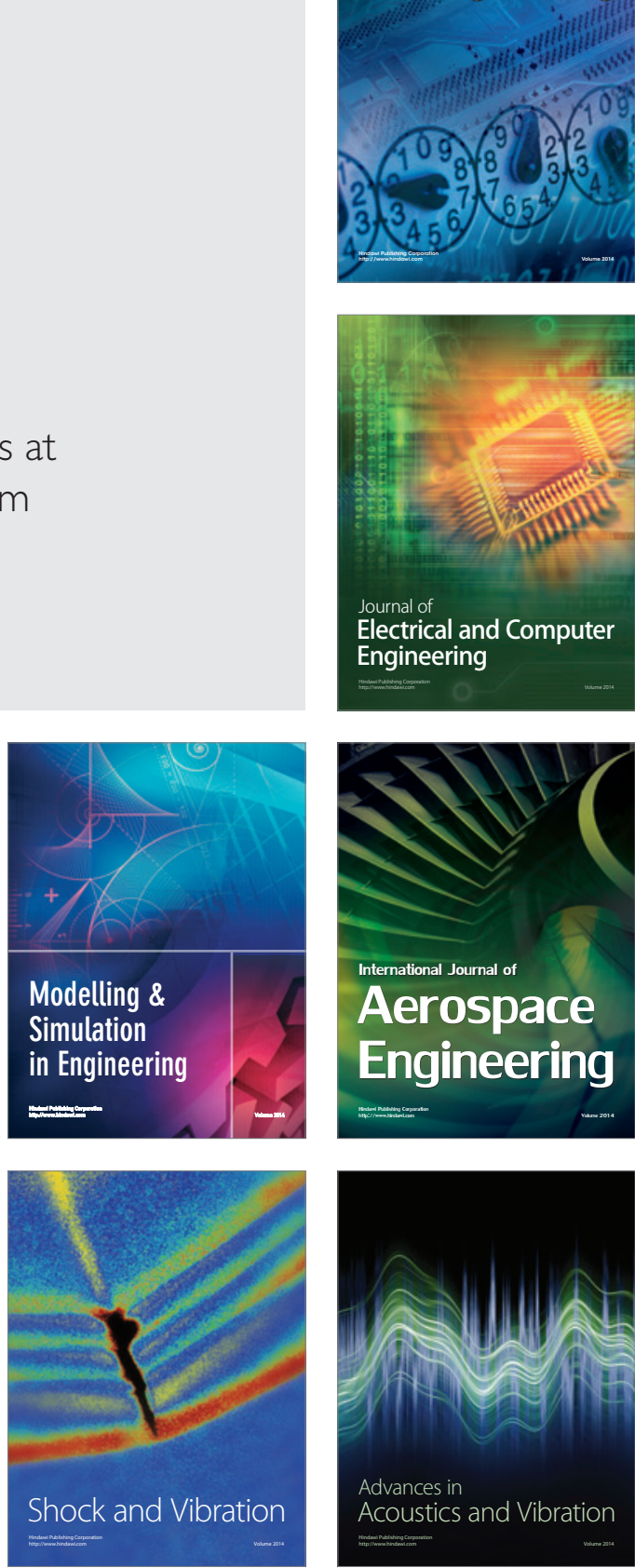